\title{
Equal fitness paradigm explained by a trade-off between generation time and energy production rate
}

Article

Accepted Version

Brown, J. H., Hall, C. A. S. and Sibly, R. M. (2018) Equal fitness paradigm explained by a trade-off between generation time and energy production rate. Nature Ecology \& Evolution, 2 (2). pp. 262-268. ISSN 2397-334X doi:

https://doi.org/10.1038/s41559-017-0430-1 Available at https://centaur.reading.ac.uk/74817/

It is advisable to refer to the publisher's version if you intend to cite from the work. See Guidance on citing.

Published version at: http://dx.doi.org/10.1038/s41559-017-0430-1

To link to this article DOI: http://dx.doi.org/10.1038/s41559-017-0430-1

Publisher: Nature

All outputs in CentAUR are protected by Intellectual Property Rights law, including copyright law. Copyright and IPR is retained by the creators or other copyright holders. Terms and conditions for use of this material are defined in the End User Agreement.

www.reading.ac.uk/centaur 
Central Archive at the University of Reading

Reading's research outputs online 
Equal fitness paradigm explained by a tradeoff between generation time and energy production rate

\author{
James H. Brown ${ }^{1 *}$, Charles A.S. Hall ${ }^{2}$ and Richard M. Sibly ${ }^{3}$ \\ ${ }^{1}$ Department of Biology, University of New Mexico, Albuquerque, NM 87131, USA; current \\ address 636 Piney Way, Morro Bay, CA 93442, USA; jhbrown@unm.edu. \\ ${ }^{2}$ Departmnent of Forest and Environmental Biology and Program in Environmental Science, \\ State University of New York - College of Environmental Science and Forestry, Syracuse NY, \\ 13210, USA; current address 26242 Montana Hwy 35, Polson, Montana 59860, USA; \\ chall@esf.edu. \\ ${ }^{3}$ School of Biological Sciences, University of Reading, Reading, UK \\ *Corresponding author
}

Most plant, animal, and microbial species of widely varying body size and lifestyle are nearly equally fit as evidenced by their coexistence and persistence through millions of years. All organisms compete for a limited supply of organic chemical energy, derived mostly from photosynthesis, to invest in the two components of fitness: survival and production. All organisms are mortal because molecular and cellular damage accumulates over the lifetime; life persists only because parents produce offspring. The equal fitness paradigm (EFP) occurs because: 1) there is a tradeoff between generation time and productive power, which have equal-but-opposite scalings with body size and temperature; smaller and warmer organisms have shorter lifespans but produce biomass at higher rates than larger and colder organisms; 2) the energy content of biomass is essentially constant, 22.4 kilojoules per gram dry body weight; and 3) the fraction of biomass production incorporated into surviving offspring is also roughly constant, $\sim \mathbf{1 0 - 5 0 \%}$. Since organisms transmit approximately the same quantity of energy per gram to offspring in the next generation, no species has an inherent lasting advantage in the struggle for existence. The EFP emphasizes the central importance of energy, biological scaling relations, and powertime tradeoffs in life history, ecology, and evolution.

Most organisms are more or less equally fit, as evidenced by the persistence of millions of plant, animal, and microbe species of widely varying size, form, and function in the earth's diverse environments. We call this the "equal fitness paradigm" (EFP). It is puzzling, because the rates and times of the life history traits that determine fitness by affecting survival and production vary by many orders of magnitude. Tiny unicellular bacteria, algae, and protists weighing a few micrograms live fast and die young, on time scales of minutes to hours, whereas large mammals and trees weighing many tonnes live on time scales of decades to centuries. How can such enormous variation in the underlying life history processes allow persistence and coexistence of so many species? The answer lies in a tradeoff in how organisms acquire, transform, and expend energy for survival and production within constraints imposed by physics and biology.

Energy and fitness. In 1905 Ludwig Boltzmann ${ }^{1}$ perceptively wrote, "The 'struggle for existence' of living beings is not for the fundamental constitutents of food ... but for the possession of the free energy obtained, chiefly by means of the green plant, from the transfer of radiant energy from the hot sun to the cold earth." Photosynthesis in plants and chemosynthesis in microbes convert solar radiation energy or inorganic chemical energy into the organic 
chemical energy of biomass. All organisms rely on this biochemical energy for two metabolic processes: 1) respiration, in which most of the organic compounds are broken down and energy is transferred to ATP to power the work of living; and 2) production, in which some of the organic compounds are repackaged into offspring biomass through growth and reproduction ${ }^{1,2,3,4,5,6,7}$. Most biomass is ultimately consumed by other organisms and passed up food chains in ecosystems.

Biophysical laws govern the stores of energy in plant, animal, and microbial biomass and the flows of energy between organisms and the environment and among organisms (Fig. 1). These laws also govern allocation of energy to the two components of fitness: survival and production. Fitness is usually measured in currencies of genes or individuals, but here we use energy as our currency of fitness as we explain below.
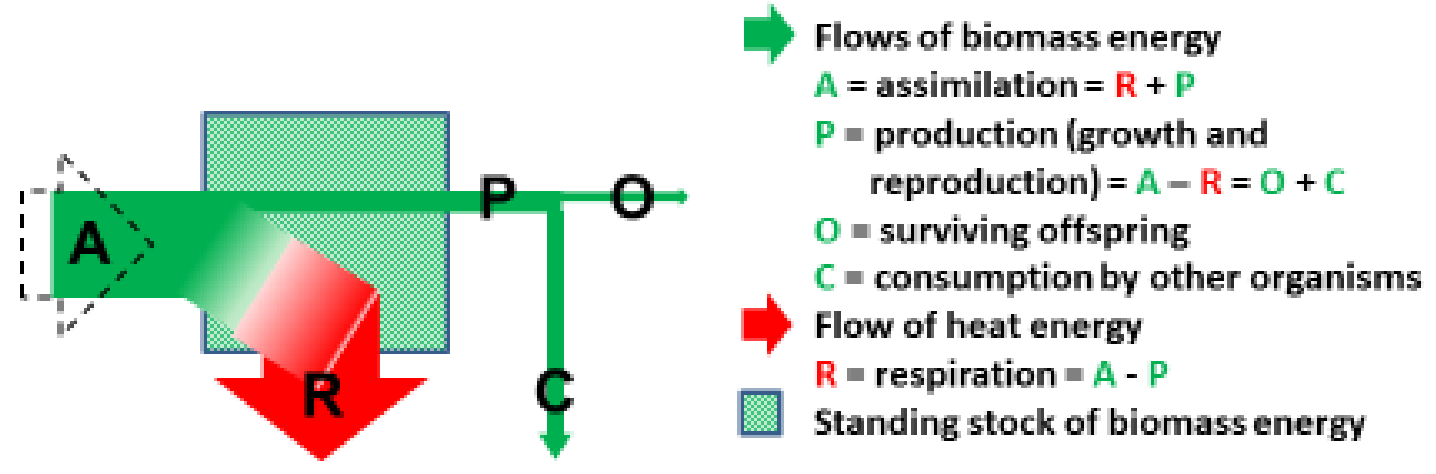

Figure 1 | Energy balance of an individual organism: A) Assimilation: chemical energy in the form of biomass is taken up from the environment by consuming other organisms (or by photosynthesis in plants); $R$ ) Respiration: most assimilated energy is metabolized to synthesize ATP, used to perform work, and ultimately released back into the environment as heat; $P$ ) Production: some assimilated biomass is repackaged into offspring via growth and reproduction; $O$ ) Surviving offspring: some of the offspring biomass survives to become an adult in the next generation; C) Consumption: some offspring die without reproducing and their biomass is assimilated by other organisms in the ecosystem.

Physical laws and biological scaling relations constrain the flows and stocks of energy in Fig. $1^{3,8,9,10,6}$. The key to the EFP is a tradeoff in how the assimilated chemical energy is allocated between the two components of fitness: survival and production. Smaller and warmer organisms produce biomass at higher rates than larger and colder organisms, but at the cost of shorter lifespans.

The rate of living: metabolic rate and survival. The respiratory metabolism that powers the work of living, and hence the survival component of fitness, also causes entropic damage that leads inevitably to aging and death. Organisms age, senesce, and die in approximate relation to their mass-specific metabolic rates, so smaller organisms with higher rates live faster and have shorter lifespans. Efforts to explain this phenomenon have led to so-called rate-of-living (ROL), disposable soma, and related concepts ${ }^{11,12,13,14}$. Recent versions of ROL theory focus on impaired molecular, cellular, and whole-organism structure and function that occurs as a consequence of oxidative metabolism. The respiration that breaks down organic compounds to synthesize ATP 
produces free-radicals and other compounds that cause damage, including telomere shortening, somatic mutations, changes in protein structure and function, and oxidative stress ${ }^{13,15,16,17}$. Some of the damage can be corrected or at least postponed by mechanisms of repair and defense, but these require expenditure of metabolic energy that could otherwise be allocated to production ${ }^{14}$. All organisms ultimately die, because imperfect repair cannot prevent aging, and also because individuals are killed by enemies and accidents.

Most of the work on ROL ideas is motivated by biomedical considerations. Largely missing is a broader framework that integrates ROL ideas into the context of the entire life history and addresses the fundamental tradeoff between survival and production. Speakman et al.

14 write "the idea that oxidative stress might underlie life-history trade-offs does not make specific enough predictions that are amenable to testing ... moreover, there is a paucity of good alternative theoretical models on which contrasting predictions might be based."

Here we present such a theoretical model and supporting empirical evidence. Our model expresses the tradeoff between the two components of fitness: generation time (lifespan) and production rate. Because all organisms are mortal, life persists only because parents produce offspring before they die.

\section{RESULTS}

A model of energy allocation to fitness. We begin by formally expressing the fitness of an individual in terms of allocation of energy to offspring in one generation. We define energetic fitness, $E$, as the mass-specific flow of biomass energy (in $\mathrm{kJ} / \mathrm{g}$ per generation) to surviving offspring, where

energetic fitness $=E=G B Q F$

and where $G$ is the generation time (in y), $B$ is the mass-specific rate of biomass production (in $\mathrm{g} / \mathrm{g} / \mathrm{y}$ ), $Q$ is the energy density of biomass (in $\mathrm{kJ} / \mathrm{g}$ ), and $F$ is the unitless fraction of the biomass production that is incorporated into surviving offspring. More specifically, $\mathrm{G}$ is the duration of the life cycle from egg to egg, so $G$ implicitly incorporates the effect of metabolic rate on survival. $B$ is the mass-specific rate of production of offspring biomass by an individual, so the sum of offspring growth and parental reproductive allocation over a lifetime divided by generation time and body mass. $F$, quantifies the fraction of the biomass produced that ends up in the offspring that survive to reproduce in the next generation. The remaining fraction $(1-F)$ is lost to prereproductive mortality and mostly consumed by other organisms (predators, parasites, pathogens, and decomposers) in the ecosystem.

Defined in this way, energetic fitness can vary among individuals and species depending on their success in leaving offspring. We proceed by making the simplifying assumption of steady state, in which the average parent produces one offspring that survives to reproduce in the next generation. This assumption, which we revisit later, is consistent with the persistence and coexistence of species over large scales of space and time. By definition fitness of all species must be equal at steady state, because there is exact replacement of parental energy in one generation; the biomass energy lost when a parent dies is replaced by the energy content of one mature offspring. But how is this EFP achieved when the species differ by orders of magnitude in survival times and production rates?

Like most biological traits, generation time, $G$, and production rate, $B$, vary with body size and temperature as quantified by the general scaling equation

$Y=Y_{0} M^{\alpha} e^{\frac{-E_{a}}{k t}}$ 
Here $Y$ is the value of the trait; the first term, $Y_{0}$, is a normalization constant; the second term gives the size dependence, where $M$ is body mass and $\alpha$ is the mass-scaling exponent; and the last term gives the temperature dependence, where $e$ is the base of the natural logarithm, $E_{a}$ is an "activation energy", $k$ is Boltzmann's constant, and $t$ is temperature in kelvin ${ }^{8,18,19}$. Recent studies have addressed the processes that determine the critical values of $\alpha$ and $E_{a}$. The massscaling exponents, $\alpha$, are typically simple multiples of $1 / 4^{15,18,20,21,22,23}$ : e.g., whole-organism metabolic rate scales as $M^{3 / 4}$ (Kleiber's law), mass-specific metabolic rate and many other rates scale as $M^{-1 / 4}$ and lifespan and most other biological times scale as $M^{1 / 4}$. These quarter-power allometries reflect geometric, physical, and biological constraints on the acquisition, distribution, transformation, and allocation of energy within the body. There is an economy of scale, so that larger organisms use less energy, generate less power, and have lower rates of respiration and production per unit mass, but they take longer to grow and reproduce and they live longer. The exponential temperature dependence reflects the kinetics of biochemical reactions and physiological processes. The value of $E_{a}$ is typically $\sim 0.65 \mathrm{eV}$, equivalent to a $Q_{10}$ of $\sim 2.5$, so rates increase and times decrease about $2 \frac{1}{2}$ times with every $10^{\circ} \mathrm{C}$ increase in temperature ${ }^{19}$.

Expanding equation (1) by substituting the scaling relations from above gives energetic fitness $=E=\left(G_{0} M^{\frac{1}{4}} e^{\frac{E_{a}}{k t}}\right)\left(B_{0} M^{-\frac{1}{4}} e^{-\frac{E_{a}}{k t}}\right) Q F$

where $G_{0}$ and $B_{0}$ are normalization constants for the scalings of generation time, $G$, and massspecific rate of biomass production, $B$, respectively, $Q$ is the energy density of biomass, and $F$ is the fraction of the biomass produced that ends up in surviving offspring. This equation is our model for energy allocation to fitness. It predicts that the EFP holds and organisms have equal energetic fitness when: 1) generation time, $G$, and production rate, $B$, have equal-but-opposite scalings with body size and temperature; 2) the energy density of biomass, $Q$, is constant; and 3 ) and the fraction, $F$, of offspring biomass that survives to reproduce in the next generation is also constant.

Testing the model. We test the model by assembling and analyzing independent data on $G, B$, and $Q$, and estimating $F$. We obtained data on allometric scalings of $G$ and $B$ from two large, recently-compiled datasets for a wide variety of animals, plants, and microbes spanning many orders of magnitude in body mass: from $10^{-12} \mathrm{~g}$ prokaryotes to $10^{8} \mathrm{~g}$ whales and trees ${ }^{26,27}$.

Generation time scales positively with body mass (Fig. 2). The exponent is statistically indistinguishable from the value of $1 / 4$ predicted for scaling of biological times ${ }^{15,22,24,25}$, so we assume that generation time scales as

$G=3.0 M^{0.25}$ 


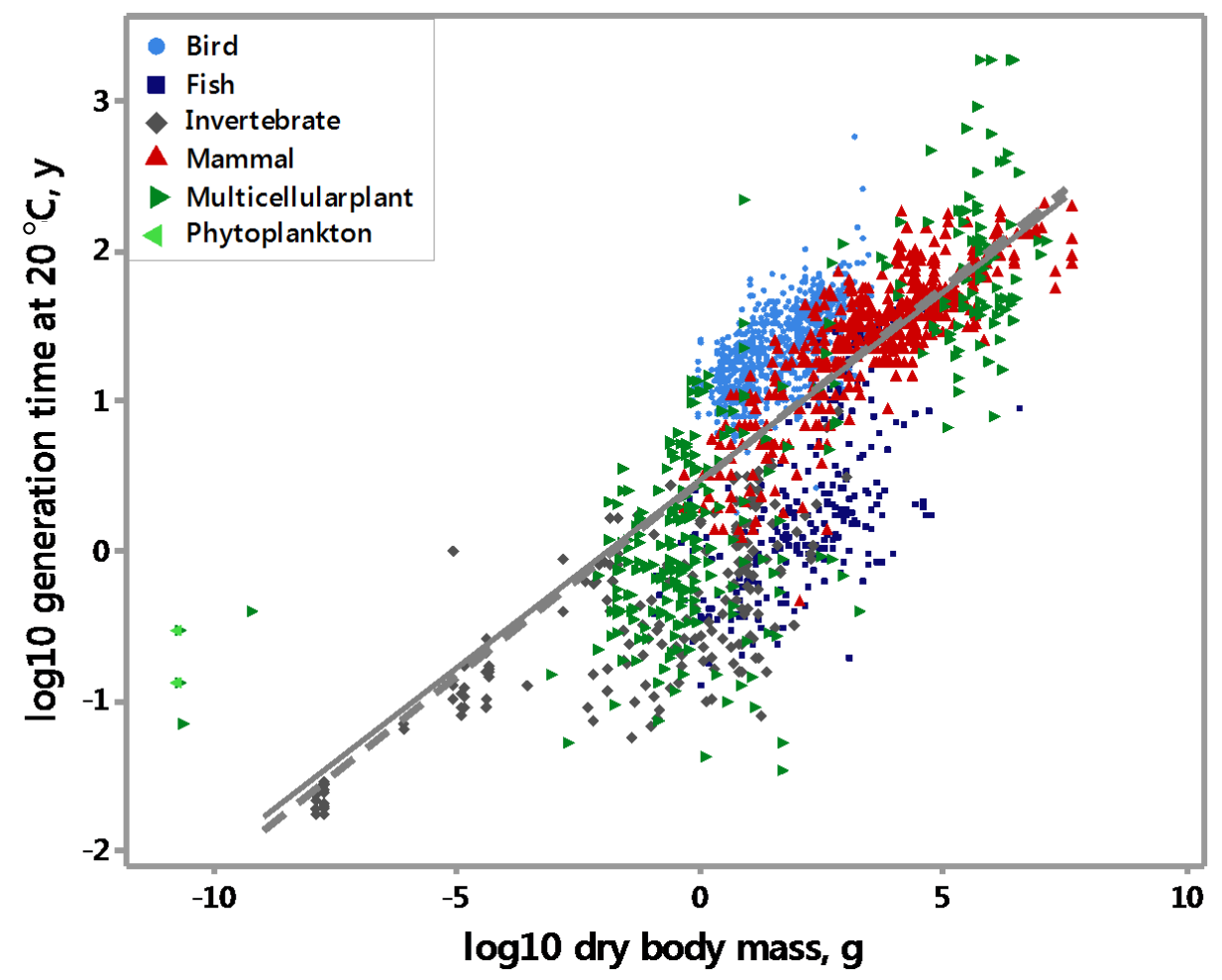

Figure 2 Generation time as a function of body mass plotted on logarithmic axes for a variety of organisms spanning more than 15 orders of magnitude in body mass. Sample sizes are: birds, 779; fish, 233; invertebrates, 195; mammals, 524; multicellular plants, 308; phytoplankton, 2. The dashed line is the fitted OLS regression, $\mathrm{y}=0.473+0.260(+/-$ SE 0.0055$) \mathrm{x}$, which gives the scaling relation $G=2.97 M^{0.26}$. We rounded and simplifed to obtain $G=3.0 M^{0.25}$, depicted by the solid line. Data were calculated from the mortality rates in McCoy and Gillooly ${ }^{26}$.

The mass-specific rate of biomass production scales negatively with body mass (Fig. 3). The scaling exponent is exactly $-1 / 4$ as predicted by metabolic theory ${ }^{24,25}$, so $B=2.54 M^{-0.25}$ 


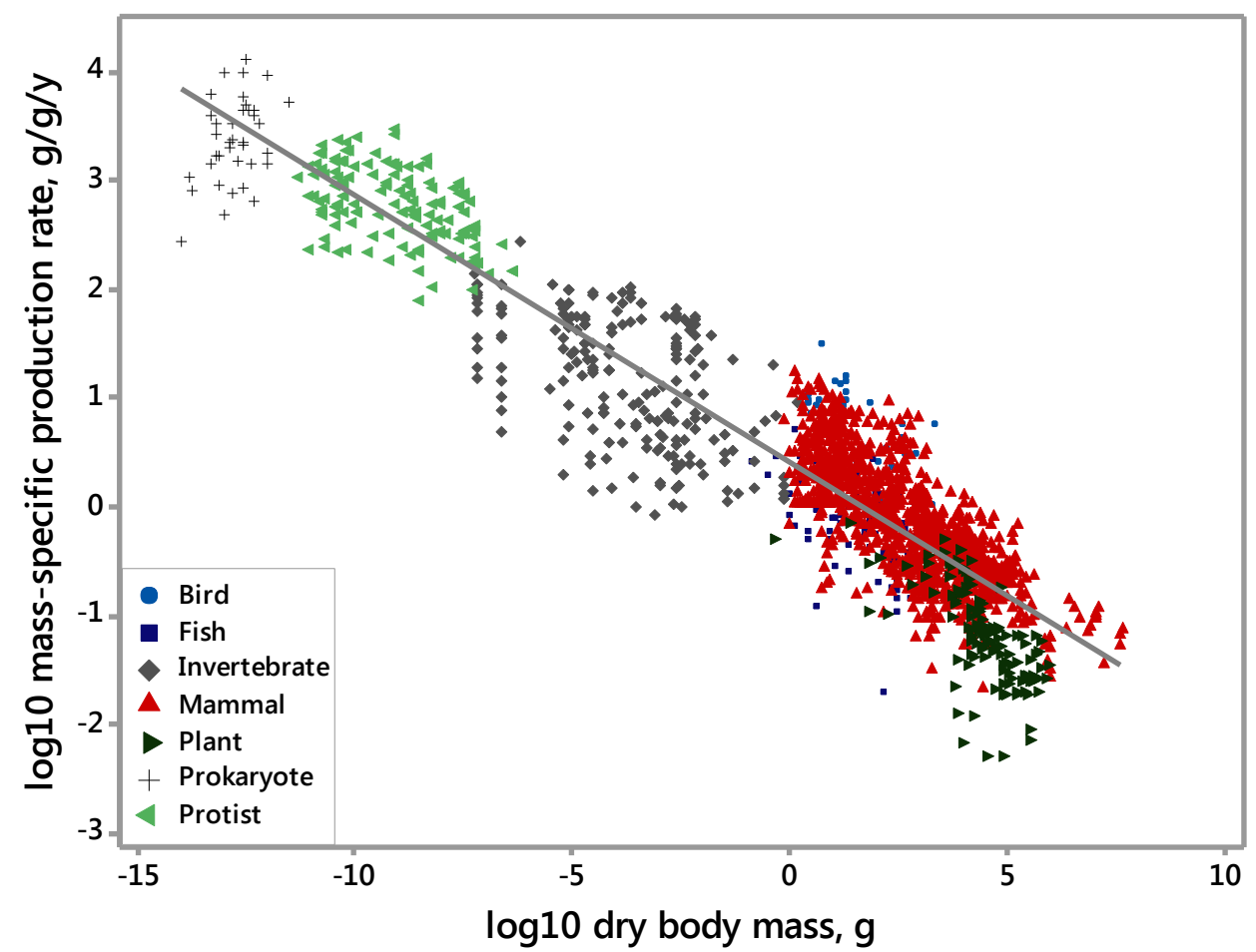

Figure 3 | Mass-specific rate of biomass production as a function of dry body mass plotted on logarithmic axes for a variety of organisms spanning more than 20 orders of magnitude in body mass. Sample sizes are: birds, 33; fish, 108; invertebrates, 197; mammals, 1061; plants, 132; prokaryotes, 37; protists, 137 . The fitted regression line $y=0.405(+/$ - SE 0.010$)-0.246(+/$ SE $0.002) \mathrm{x}$ gives the scaling relation $B=2.54 M^{-0.25}$. Data were calculated from the compilation of Hatton et al. ${ }^{27}$.

The energy density of biomass ${ }^{28}$ varies less than two fold, between constraint boundaries due to chemical composition (Fig. 4; see methods). Hereafter we assume that $Q$ is nearly constant, $\sim 22.4 \mathrm{~kJ} / \mathrm{g}$ ash-free dry weight. This value is intermediate between the $23 \mathrm{~kJ} / \mathrm{g}$ and 22 $\mathrm{kJ} / \mathrm{g}$ reported in the literature ${ }^{8,29}$. 


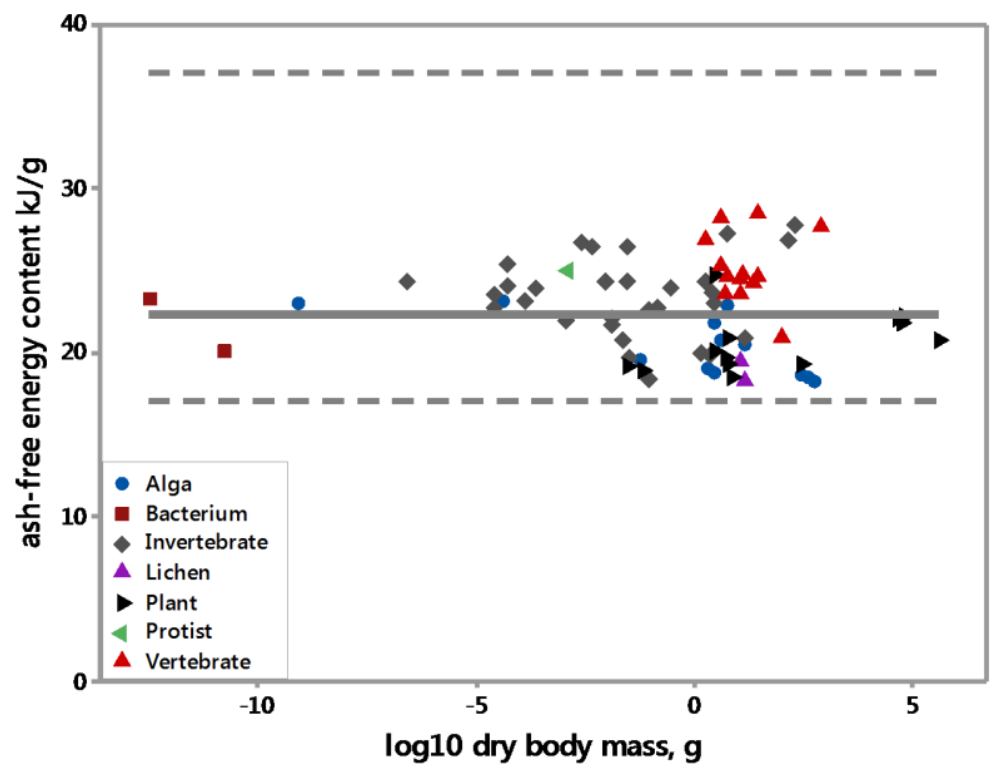

Figure 4 | Ash-free energy content of dry biomass, $Q$, as a function of body mass for a variety of animals, plants, and microbes spanning more about 18 orders of magnitude in body mass. Sample sizes are: algae, 13; bacteria, 2; invertebrates, 30; lichen, 2; plants, 13; protists, 1; vertebrates, 13. OLS regression gave no significant scaling relation, $y=22.38-0.087(+/-\mathrm{SE}$ 0.10). Data are from the compilation of Cummins and Wuychek ${ }^{28}$. The solid line indicates the mean value, $22.4 \mathrm{~kJ} / \mathrm{g}$, and the dashed lines indicate the upper and lower bounds of 17 and 37 $\mathrm{kJ} / \mathrm{g}$ in the unlikely case if the body were composed entirely of carbohydrates and proteins or entirely of lipids.

The value of $F$, the fraction of production that is passed on to the next generation in surviving offspring, was estimated by re-arranging equation (1), assuming $E=22.4 \mathrm{~kJ} / \mathrm{g}$, and substituting equations (4) and (5) from above, to obtain

$F=\frac{E}{G B Q}=\frac{22.4}{(22.4) G B}=\frac{1}{G B}=\frac{1}{\left(3.0 M^{0.25}\right)\left(2.54 M^{-0.25}\right)}=0.13$

Because this calculation gives no information on variation, we also estimated $F$ for two species of contrasting body size and complexity: 1) a hypothetical microscopic unicellular microbe that reproduces by mitotic division, so it doubles its mass and energy content, divides, and at steady state one of the cells survives to replace the parent, so $F=0.5$; and 2) a sockeye salmon weighing $2,700 \mathrm{~g}$, where $F \approx 0.15$ (methods). We conclude that $F$ varies from about 0.1 to 0.5 , which like the variation in $Q$ (above and methods) is miniscule over the more than 20 orders-ofmagnitude variation in body mass. Hereafter we assume that $F \approx 0.13$, independent of body size and temperature.

Our model for energetic fitness, obtained by rearranging equation (6) and substituting empirical values to parameterize equations (1) and (3), is energetic fitness $=E=G B Q F=\left(2.54 M^{-0.25}\right)\left(3.0 M^{0.25}\right) Q F=$ $(2.54)(3.0)(22.4)(0.13)=22.19 \mathrm{~kJ} / \mathrm{g}$ per generation

So at steady state and within rounding errors, all organisms replace themselves in one generation with nearly the same quantity of energy, $Q \approx 22.4 \mathrm{~kJ} / \mathrm{g}$. The equal-but-opposite scalings of $G$ and $B$ in equations (4) and (5) quantify the fundamental life history tradeoff between production and 
survival, and therefore between power ( $B Q$ in watts per gram) and time ( $G$ in years). The effect of smaller body mass in increasing mass-specific power is exactly offset by its effect in decreasing generation time. Because biological times and rates also have equal-but-opposite scalings with temperature (equation (3)), the effect of temperature is also offsetting ${ }^{19,23}$ : higher temperature increases production rate but decreases generation time by an equal amount. If sufficient data is compiled, it will be straightforward to test this prediction.

\section{DISCUSSION}

The model of energy allocation. The complete empirically-parameterized model for the EFP is shown diagrammatically in Fig. 5. This model is very general and robust; the tradeoff between generation time and productive power applies over the entire 22 orders of magnitude variation in body mass of living things - from $10^{-13}$-gram microbes to $10^{9}$-gram whales and trees - and over the biologically relevant temperature range from $0-40^{\circ} \mathrm{C}$. This model is not intended to replace or conflict with expressions for fitness couched in genetic or life history terms, but to supplement and complement those formulations. Metabolism is equally as important as genetics, because organisms pass on energy as well as genes to the next generation, and because the organic chemicals produced by biosynthesis are the only source of the metabolic energy that sustains life $\mathrm{e}^{1,2,3,4,6,7,9,24}$.

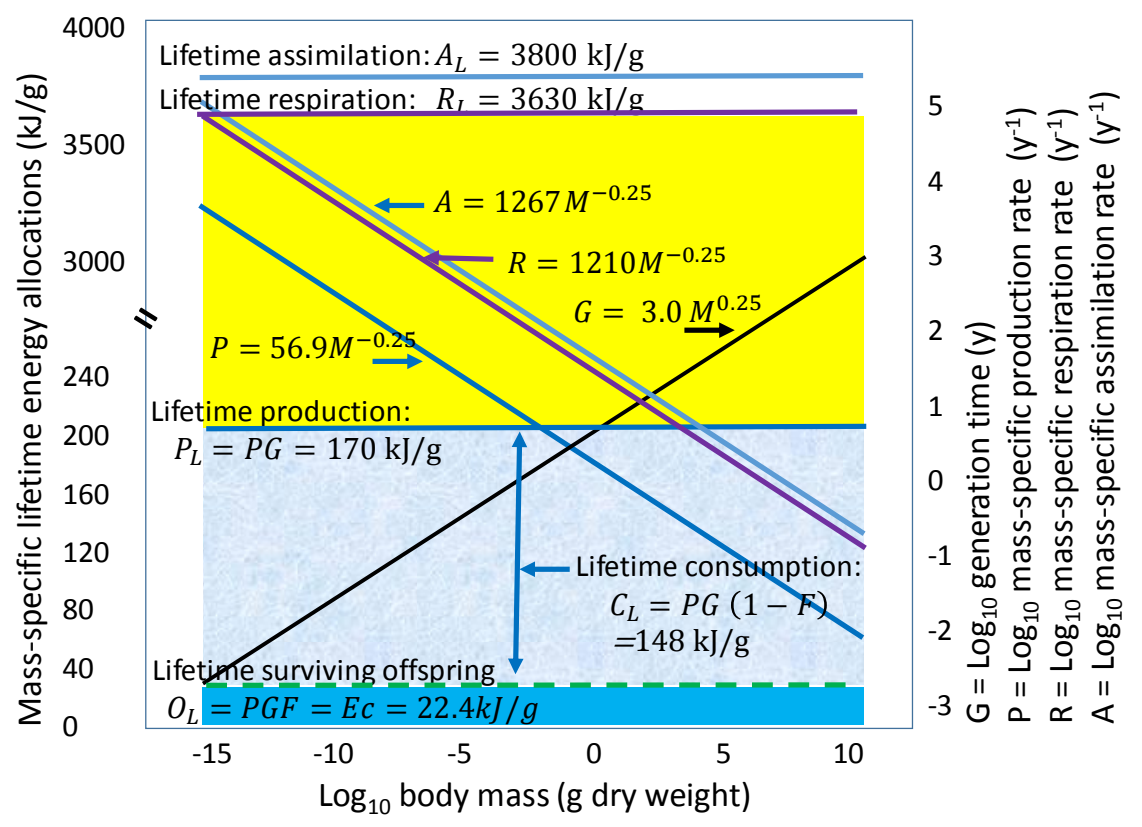

$$
\begin{aligned}
& \mathrm{F}=\mathrm{O} / \mathrm{P} \approx 0.13 \\
& \mathrm{~T}=\mathrm{P} / \mathrm{A}=0.045
\end{aligned}
$$

Figure 5 | The model for EFP parameterized with data from the text. Shown are the scalings with body mass, $M$, of generation time, $G$, and the mass-specific rates of assimilation, $A$, respiration, $R$, and energy production, $P$ (where $P=B Q$ ). Also shown are the total lifetime energy expenditures for assimilation, $A_{L}$, respiration, $R_{L}$, production, $P_{L}$, and surviving offspring, $O_{L}$. The EFP arises from the special conditions shown here: 1$)$ generation time, $G$, and mass-specific rate of biomass production, $P$, have equal-but-opposite scalings with body mass, $M$; 2) $Q$, the energy content of biomass is constant; and 3) $F$, the fraction of energy incorporated into the surviving offspring, $O$, is also constant. Because $A, R, P$, and $O$ scale with body mass with the 
same exponent (-0.25), the lifetime allocations per gram per generation and the ratios $O / P=F$ and $P / A=T$ are also approximately constant.

Figure 5 shows how the energy budget in Fig. 1 depends on the precise quarter-power scalings of biological times and rates with body mass. Generation time scales with an exponent of 1/4 and mass-specific rates of assimilation and production with exactly opposite exponents of -1/4 (equations (3-7)). So lifetime expenditures (horizontal lines in Fig. 5) are nearly constant across many orders of magnitude variation in body size. The fact that the rates all scale with the same exponent (-1/4) has the additional consequence that their ratios must also be constant and independent of body size (and temperature). Above we estimated that $F=O / P$, the fraction of energy production that is incorporated into surviving offspring, $\approx 0.13$. We also estimated the trophic transfer efficiency, $T=P / A$, the fraction of assimilated energy repackaged into offspring biomass (see methods). For sockeye salmon $T \approx 0.18$ based on direct data; for multiple species of mammals $T \approx 0.045$ based on allometric scaling relations. These values bracket the $\sim 0.10=10 \%$ often reported in ecology textbooks as a rough average across diverse taxa and ecosystems. Our values are consistent with evidence that endothermic mammals and birds, which spend a substantial portion of their energy budget on respiration to maintain a high and relatively constant body temperature, are less efficient producers than most other (ectothermic) organisms $s^{5,9,30,31}$.

From the above it follows that the vast majority of the energy assimilated over the life cycle is expended on respiration to power the cost of living and is ultimately dissipated to heat. Only a small fraction ( $\sim 18 \%$ in salmon, $\sim 4.5 \%$ in mammals) is converted into biomass, and only a small fraction of that is passed on to surviving offspring ( $14 \%$ of production or $\sim 2.5 \%$ of total lifetime assimilation in salmon; $\sim 4.5 \%$ of production or $\sim 0.6 \%$ of total assimilation in mammals).

Accounting for the variation. We regard the empirical validation of the model as preliminary. In particular, the data in Figs. 2 and 3 exhibit considerable variation around the regression lines. Many studies of macroecology and biological scaling necessarily rely on "messy data" and statistical relations over many orders of magnitude and wide scales of space, time, and biodiversity. General patterns, such as size-scaling relations, can be quantified with considerable precision despite substantial variation in individual values (see methods for the example of the energy density of biomass). Moreover, some of the apparent variation may be due to errors or lack of standardization in the empirical measurements.

When the data are accurate, however, the variation presumably reveals either flaws in the theory or additional important factors not included in the model. There is real body sizeindependent variation between individual species in in data values, and real differences between taxonomic and functional groups in normalization constants for allometric scaling relations. For example, primates have similar metabolic rates as other mammals of similar body size, but they have substantially longer lifespans and generation times and as our model predicts they are correspondingly less productive than most mammals ${ }^{32}$ (similarly for birds vs mammals ${ }^{13}$ ).

We encourage additional work to evaluate the theory and empirical evidence presented above. An important issue is how well the energetic framework and specific model apply to microbes. In unicellular eukaryotes, mass-specific metabolic rate is approximately invariant with body mass rather than scaling as $M^{-1 / 4}$ as predicted by Kleiber's rule ${ }^{33}$. But larger cells take longer to grow and divide ${ }^{33,34}$, so generation time scales as $M^{1 / 4}$ and mass-specific rate of 
biomass production scales as $M^{-1 / 4}$ as predicted by equations (4) and (5). Prokaryotes may be more problematic. Recent studies suggest that some energetic constraints are near-universal characteristics of life $\mathrm{f}^{34,35}$. But the finding that mass-specific metabolic rate scales positively with body mass in prokaryotes ${ }^{33}$ raises questions about the scalings of generation time and production rate.

EFP differs from LRE, $\boldsymbol{R}_{\max }$, and MPP. Life history theory has addressed tradeoffs between reproduction and survival ${ }^{36,37,38}$. Most relevant here are two studies showing that lifetime reproductive effort $(\mathrm{LRE})^{39}$ and the related average lifetime reproductive rate $\left(\mathrm{R}_{\max }\right)^{40}$ are approximately constant across organisms despite wide variation in body sizes and rates of production and mortality. Like many treatments of fitness, LRE and $\mathrm{R}_{\max }$ focus on reproduction. Our analysis highlights the additional importance of growth. Production includes both growth, biomass energy accumulated by offspring independent of their parents, and reproduction, energy in the form of gametes, intra-uterine nutrition, and food supplied to offspring by parents. Growth accounts for the vast majority of energy production in many species; they produce large numbers of miniscule offspring, which grow to adult size fueled entirely by their own assimilation and growth.

The EFP is also reminiscent of the "maximum power principle" (MPP) $)^{2,41,42,43}$. The idea that individuals which generate more power should have more energy to invest in survival and reproduction was initially attractive, because it appeared to account for the increase in body size over evolutionary history ${ }^{44}$. Large, slow trees and mammals are more powerful on a wholeorganism basis, but they are no fitter than small, fast bacteria, algae, and protists that evolved earlier but still persist as the most abundant life forms on earth. The EFP explicitly includes power as a component of fitness, but only as it trades off with generation time.

Revisiting the steady-state assumption. Steady state is a reasonable assumption when it is appropriate to average over many individuals and generations. At smaller scales, however, individuals and populations are rarely at steady state, the EFP may not hold, and $E$ may deviate substantially from $22.4 \mathrm{~kJ} / \mathrm{g}$ per generation.

Most relevant to any consideration of fitness, the steady state assumption is violated when natural selection occurs. Fitter individuals more than just replace themselves; they leave more offspring, genes, and energy in subsequent generations, so $E>22.4 \mathrm{~kJ} / \mathrm{g}$ per generation. Heritable traits that increase $E$ by increasing $G, B$, or $F$ will tend to increase in frequency due to natural selection. But as individuals differentially replace themselves and populations grow, their fitness advantage is only temporary. Eventually they reach limits, lose their advantage, and return back toward the steady state where fitness is equal. This occurs in ecological time, because finite environmental conditions prevent indefinite population growth (the "ecological compensation" of Sibly and $\mathrm{Calow}^{44}$ ). It also occurs in evolutionary time because of the "Red Queen" phenomenon ${ }^{4,46}$. Total ecosystem respiration consumes nearly all primary production ${ }^{7,46}$, so there is "a zero-sum game for energy"; any fitness advantage or increase in one species is soon checked by ecological interactions and coevolution with other species. Because the steady state assumption was temporarily violated and fitness was not always exactly equal, the history of life on earth has witnessed many origins and extinctions of species and much turnover in taxonomic and functional groups. But life has persisted and diversified because the fitnesses of the millions of plant, animal, and microbe species were very nearly equal. 
The steady state assumption has been violated to achieve historic increases in production of domestic plants and animals. Agriculturalists have used artificial selection, husbandry, and massive inputs of fossil fuel energy to alter environment, physiology, and behavior to increase the efficiency of conversion of assimilated energy into biomass. Results have been spectacular, achieving $P / A \approx 33-50 \%$ for factory-farmed turkeys, chickens, hogs, and fish ${ }^{47}$, compared to trophic exchange efficiencies of $T=P / \mathrm{A} \leq 20 \%$ for wild animals (above). A corollary is that these increases in biomass production for human consumption have been achieved by reduced energy allocation to traits that enhance survival and production in the wild.

The importance of the constants $Q$ and $\boldsymbol{F}$. Our analysis emphasizes the importance of a nearuniversal biological constant, the energy density of biomass, $Q \approx 22.4 \mathrm{~kJ} / \mathrm{g}$ ash free dry mass. This value reflects the physical chemistry of organic molecules and the fact that these molecules are the only source of the metabolic energy that sustains life.

The value of $F$, the fraction of production that is incorporated into the offspring that survive to breed in the next generation, also warrants increased attention. The relative constancy of $F$ contrasts with the enormous variation in number of offspring produced per lifetime: from millions of tiny larvae or seeds in some large clams, fish, and trees, to a few large babies in some birds and mammals that provide extensive parental care. Our findings suggest that relationships between growth and mortality of offspring are constrained so that $F$ is constrained within narrow bounds, $\sim 0.1-0.5$ : approximately constant relative to the many orders of magnitude variation in body size, generation time, and production rate. The broader implication is that competition for essential biochemical energy has resulted in the evolution and persistence of species that allocate similar fractions of assimilation to production and pass similar fractions of production on to offspring in the next generation. These corollaries of the EFP reflect the powerful role of Red Queen energy-based processes in the evolution of life histories and the origin and persistence of biodiversity.

Other mechanisms underlying biodiversity. The EFP is necessary but not sufficient to account for the origin and persistence of the millions of species living on earth. The body size and temperature dependence in our model can account for the persistence of different-sized organisms in environments of varying temperature. But the EFP cannot account for the coexistence of multiple species of the same size or for the effects of other environmental variables, such as water or nutrients. Ecologists have traditionally used concepts of multidimensional ecological niches ${ }^{48}$ and energy return on investment ${ }^{49,50}$ to explain the effects of abiotic conditions and interspecific interactions on the abundance, distribution, and diversity of species. To the extent that they can account for stable coexistence, these concepts implicitly assume the EFP.

Broader implications. The power-time tradeoff and its expression in the equal-but-opposite scalings of generation time and production rate are consequences of biophysical laws. The geometric, physical, and biological underpinnings for these laws have received considerable recent attention. The inverse correlation between metabolic rate and lifespan that has been a focus of rate of living (ROL) studies is a biological manifestation of the Second Law of Thermodynamics. The oxidative respiration that generates ATP and powers the work of living also generates compounds that cause damage at molecular, cellular, and organismal levels. This entropic damage accumulates over the lifespan and leads inevitably to senescence and death ${ }^{12,14}$. 
Life persists despite this damage, because the production of offspring replaces the mortality of parents. Somehow, in the process of reproduction, entropic damage is repaired, the clock of aging is reset, and there is a temporary, one-generation reprieve from the effects of the Second Law. The thermodynamic underpinnings for this amazing rejuvenation remain to be elucidated.

The EFP and our model for energetic fitness highlight the role of energy as the ultimate limiting resource in ecology and evolution ${ }^{1,2,4,7,46}$. All living things compete for the energy captured in organic molecules by biosynthesis. At the levels of ecosystems and the biosphere, nearly all of the chemical energy captured in primary production is ultimately consumed and degraded to heat in respiration. The result is a zero sum competitive game in which any species that temporarily acquires a disproportionate share has only a short-lived advantage because of competition and coevolution with other species. The EFP is a necessary condition for the origin and persistence of the earth's spectacular biodiversity. The millions of contemporary species are survivors of unbroken chains of individual organisms that acquired a sufficient share of organic chemical energy to leave surviving offspring over the countless generations since the origin of life on earth 4 billion years ago.

\section{METHODS}

Estimating the scalings of $\boldsymbol{G}$ and $\boldsymbol{P}$ with body mass. Data on generation times and body sizes came from McCoy and Gillooly ${ }^{26}$, who collected and analyzed data on natural mortality and body mass of over 1,500 species. Phytoplankton data were included only if they were for a single species (unused data were for entire communities or ecosystems). Mortality rates were corrected for temperature to $20^{\circ} \mathrm{C}$ using the Arrhenius function with activation energy $=0.65 \mathrm{kV}$.

Specifically, corrected mortality rate $=Z e^{\frac{0.65}{k}\left(\frac{1}{t}-\frac{1}{293.15}\right)}$, where $\mathrm{Z}$ is uncorrected mortality rate, $\mathrm{t}$ is temperature in kelvin and $\mathrm{k}$ is Boltzmann's constant $=0.00008617 \mathrm{eV}$. Generation time at $20^{\circ} \mathrm{C}$ was calculated as the reciprocal of corrected mortality rate.

Data on rates of biomass production and body sizes were obtained from Hatton et al. ${ }^{27}$, who compiled data on rates of whole-organism biomass production and wet body mass for over 1,000 species. To analyze their data and prepare Fig. 3, we expressed their data as mass-specific production rate and converted to units of dry body mass assuming dry mass = (wet mass)/4).

Estimating the values of $\boldsymbol{Q}, \boldsymbol{F}$, and $\boldsymbol{T}$. We used two lines of evidence to estimate Q, the energy density of biomass, and to test the prediction that $Q$ is nearly constant:

1) The constraint that biomass is composed of a mixture of carbohydrates, proteins, and lipids bounds the scaling of $Q$. The energy content of carbohydrates and proteins is $\sim 17 \mathrm{~kJ} / \mathrm{g}$ and of lipids is $\sim 37 \mathrm{~kJ} / \mathrm{g}$ of dry biomass. The proportions of these compounds vary somewhat within individuals and among species. But the energy content of all organisms must lie between these limits, which differ by a maximum possible value of $\sim 20 \mathrm{~kJ} / \mathrm{g}$ for the unrealistic cases of an organism composed entirely of carbohydrates and proteins or entirely of lipids. So the slope over 22 orders of magnitude variation in body size can be at most an infinitesimally small $20 \times 10^{-22}$ $\mathrm{kJ} / \mathrm{g} / \mathrm{g}$.

2) There are empirical measurements of energy density for a wide variety of organisms. The best measure of $Q$ is $\mathrm{kJ} / \mathrm{g}$ of ash-free biomass as determined by direct calorimetry. We obtained data from Cummins and Wuychek ${ }^{28}$ for several taxonomic and functional groups from tiny microbes to vertebrates and large plants and used independent sources to estimate dry body mass. The data are in SI Table 2 and are plotted in Fig. 4. 
We estimated the value of $F$, the fraction of production that is passed on to the next generation in surviving offspring, in three ways:

1) We assumed $E=22.4 \mathrm{~kJ} / \mathrm{g}$ and re-arranged equation (1) to obtain equation (6). This calculation did not provide any information on variation in $F$.

2) We estimated $F$ for a microscopic unicellular microbe that reproduces by mitotic division: in one generation an individual doubles its mass and energy content, divides, and at steady state one of the cells survives to replace the parent, so $F=0.5$.

3) We estimated $F$ using Brett's ${ }^{51}$ data for the Babine Lake population of sockeye salmon to compile an energy budget over the entire life cycle from egg to breeding adult. Over its lifetime an average individual produced $P \approx 55000 \mathrm{~kJ}$ of biomass in body growth and gametes to leave one surviving breeding offspring in the next generation with an energy content of $O \approx 8000 \mathrm{~kJ}$, so $F=O / P \approx 8000 / 55000 \approx 0.145 \approx 14.5 \%$.

We estimated the value of $T=P / A$, the fraction of assimilated energy passed through as biomass energy production or the trophic exchange efficiency, for both salmon and mammals. Brett' $^{51}$ data for sockeye salmon over the entire life cycle give cumulative $P \approx 55000 \mathrm{~kJ}$ and cumulative respiration $R \approx 254000 \mathrm{~kJ}$. Total assimilation, $A=P+R$, so $T=P /(P+R) \approx$ $55000 /(55000+254000) \approx 0.178 \approx 18 \%$. To estimate $T$ for mammals we used data from Nagy's ${ }^{52}$, who compiled data from studies that used doubly-labeled water to measure respiration rates of free-living mammals in the field. We converted to dry body mass, assuming dry mass $=$ (wet mass)/4 to obtain the mass-specific scaling relation $R=1210 M^{-0.27}$, where $R$ is in $\mathrm{kJ} / \mathrm{g} / \mathrm{y}$, $M$ is dry body mass in $\mathrm{g}$, and the exponent is not significantly different from $-1 / 4$. Rounding and combining this with the scaling of mass-specific energy production $\left(P=2.54 M^{-0.25}\right.$; Fig. 3), from above, we estimated $A=R+P \approx\left(1210 M^{-0.25}\right)+\left(56.9 M^{-0.25}\right) \approx 1267 M^{-0.25}$ and $T=$ $P / A \approx \frac{56.9 M^{-0.25}}{1267 M^{-0.25}} \approx 0.045$.

The data used in Figs. 2 and 4 are presented as Excel files in SI Tables 1 and 2.

${ }^{51}$ Brett, J.R. Life energetics of sockeye salmon, Oncorhynchus nerka. In Behavioral energetics: the cost of survival in vertebrates (eds Aspey, W.P. \& Lustick, S.I.) 2, 29-63 (Ohio State University Press, 1983).

${ }^{52}$ Nagy, K.A. Field metabolic rate and body size. J. Exper. Biol. 208, 1621-1625 (2005).

\section{Data Availability Statement}

All data presented and analysed in this paper are available either in the cited references or in SI Tables 1 and 2.

\section{Acknowledgements}

We thank the following individuals for helpful discussions of ideas and/or comments on the manuscript: G Boyle, JR Burger, K Cummins, J Damuth, BJ Enquist, JF Gillooly, R Hengeveld, C Jordan, A Kodric-Brown, C Levitan, JG Okie, and D Storch.

\section{Author Contributions}

All three co-authors contributed to all aspects of the work.

\section{Declaration of Financial Competing Interests}

The authors declare no competing financial interests. 


\section{References}

1. Boltzmann, L. The Second Law of Thermodynamics (Populare Schriften. Essay No. 3 (Address to Imperial Academy of Science in 1886)). Reprinted in English in: Theoretical Physics and Philosophical Problems, Selected Writings of L. Boltzmann (D. Riedel, Dordrecht, 1905).

2. Lotka, A.J. Contribution to the energetics of evolution. Proc. Natl Acad. Sci. USA 8, 147-151 (1922).

3. Brody, S. Bioenergetics and growth (Reinhold Publishing, 1945).

4. Van Valen, L. Evolution as a zero-sum game for energy. Evolutionary Theory 4, 289-300 (1980).

5. McNab, B.K. The physiological ecology of vertebrates: a view from energetics (Cornell Univ. Press, 2002).

6. Humphries, M.M. \& McCann, K.S. Metabolic ecology. J. Animal Ecol. 83, 7-19 (2014).

7. Judson, O. P. The energy expansions of evolution. Nature Ecol. Evol. 1, 0138 (2017).

8. Peters, R.H. The ecological implications of body size (Cambridge Univ. Press, 1983).

9. Karasov, W.H. \& del Rio, C.M. Physiological ecology: how animals process energy, nutrients, and toxins. (Princeton Univ. Press, 2007).

10. Sibly, R.M., Grimm, V., Martin, B.T., Johnston, A.S., Kułakowska, K., Topping, C.J., Calow, P., Nabe-Nielsen, J., Thorbek, P. \& DeAngelis, D.L. Representing the acquisition and use of energy by individuals in agent-based models of animal populations. Methods Ecol. \& Evol. 4, 151-161 (2013).

11. Pearl, R. The rate of living (Knopf, 1928).

12. Harman, D. Aging: a theory based on free radical and radiation chemistry. J. Gerontol. 11, 298-300 (1956).

13. Speakman, J.R. Body size, energy metabolism and lifespan. J. Exp. Biol. 208, pp.1717-1730 (2005).

14. Speakman, J.R., Blount, J.D., Bronikowski, A.M., Buffenstein, R., Isaksson, C., Kirkwood, T.B., Monaghan, P., Ozanne, S.E., Beaulieu, M., Briga, M. \& Carr, S.K. Oxidative stress and life histories: unresolved issues and current needs. Ecol. \& Evol. 5, 5745-5757 (2015).

15. Calder, W.A. Size, function, and life history. (Harvard Univ. Press, 1984).

16. Martin, A.P. \& Palumbi, S.R. Body size, metabolic rate, generation time, and the molecular clock. Proc. Natl Acad. Sci. USA 90, 4087-4091 (1993).

17. Atanasov, A.T. The linear allometric relationship between total metabolic energy per life span and body mass of poikilothermic animals. Biosystems 82, 137-142 (2005).

18. Schmidt-Nielsen, K. Scaling: why is animal size so important? (Cambridge Univ. Press, 1984).

19. Gillooly, J.F., Brown, J.H., West, G.B., Savage, V.M. \& Charnov, E.L. Effects of size and temperature on metabolic rate. Science 293, 2248-2251 (2001).

20. Kleiber, M. Body size and metabolism. Hilgardia 6, 315-353 (1932).

21. West, G.B., Brown, J.H. \& Enquist, B.J. A general model for the origin of allometric scaling laws in biology. Science 276, 122-126 (1997).

22. Lindstedt, S.L. \& Calder III, W.A. Body size, physiological time, and longevity of homeothermic animals. Quart. Rev. Biol. 56, 1-16 (1981).

23. Gillooly, J. F., Charnov, E. L., West, G. B., Savage, V. M., \& Brown, J. H. Effects of size and temperature on developmental time. Nature 417, 70-73 (2002). 
24. Brown, J.H., Gillooly, J.F., Allen, A.P., Savage, V.M. \& West, G.B. Toward a metabolic theory of ecology. Ecology 85, 1771-1789 (2004).

25. Sibly, R.M., Brown, J.H. \& Kodric-Brown, A. eds. Metabolic ecology: a scaling approach (John Wiley-Blackwell, 2012).

26. McCoy, M.W. \& Gillooly, J.F. Predicting natural mortality rates of plants and animals. Ecol. Lett. 11, 710-716 (2008), 12, 731-733 (2009).

27. Hatton, I.A., McCann, K.S., Fryxell, J.M., Davies, T.J., Smerlak, M., Sinclair, A.R.E. \& Loreau, M. The predator-prey power law: Biomass scaling across terrestrial and aquatic biomes. Science 349, 1070-1070 (2015).

28. Cummins, K.W. and Wuychek, J.C. Calorific equivalents for studies in ecological energetics. Intl Assoc. Theor. \& Appl. Limnol. 18, 1-158 (1967).

29. Brey, T., Rumohr, H. \& Ankar, S. Energy content of macrobenthic invertebrates: general conversion factors from weight to energy. J. Exp. Marine Biol. \& Ecol. 117, 271-278 (1988).

30. Humphreys, W.F. Production and respiration in animal populations. J. Animal Ecol. 48, 427453 (1979).

31. May, R.M. Production and respiration in animal communities. Nature 282, 443-444 (1979).

32. Charnov, E.L. \& Berrigan, D. Why do female primates have such long lifespans and so few babies? Or life in the slow lane. Evol. Anthro. 1, 191-194 (1993).

33. DeLong, J.P., Okie, J.G., Moses, M.E., Sibly, R.M. \& Brown, J.H. Shifts in metabolic scaling, production, and efficiency across major evolutionary transitions of life. Proc. Natl Acad. Sci. USA 107, 12941-12945 (2010).

34. Lynch, M. \& Marinov, G.K. The bioenergetic costs of a gene. Proc. Natl Acad. Sci. USA 112, 15690-15695 (2015).

35. Corkrey, R., McMeekin, T.A., Bowman, J.P., Ratkowsky, D.A., Olley, J. \& Ross, T. The biokinetic spectrum for temperature. PloS one 11, p.e0153343 (2016).

36. Williams, G.C. Natural selection, the costs of reproduction, and a refinement of Lack's principle. Amer. Natur. 100, 687-690 (1966).

37. Stearns, S.C. The evolution of life histories (Oxford Univ. Press, 1992).

38. Charnov, E.L. Life history invariants: some explorations of symmetry in evolutionary ecology (Oxford Univ. Press, 1993).

39. Charnov, E. L., Warne, R., \& Moses, M. Lifetime reproductive effort. Amer. Natur. 170, E129-E142 (2007).

40. Ginzburg, L.R., Burger, O. \& Damuth, J. The May threshold and life-history allometry. Biol. Lett. 6, 850-853 (2010).

41. Odum, H.T. \& Pinkerton, R.C. Time's speed regulator: the optimum efficiency for maximum power output in physical and biological systems. Amer. Sci. 43, 331-343 (1955).

42. Odum, H.T. Environment, power, and society (Wiley-Interscience, 1971).

43. Hall, C.A.S. Maximum power: the ideas and applications of H.T. Odum (Univ. Press Colorado, 1995).

44. Payne, J.L., Boyer, A.G., Brown, J.H., Finnegan, S., Kowalewski, M., Krause, R.A., Lyons, S.K., McClain, C.R., McShea, D.W., Novack-Gottshall, P.M. \& Smith, F.A. Two-phase increase in the maximum size of life over 3.5 billion years reflects biological innovation and environmental opportunity. Proc. Natl Acad. Sci. USA 106, 24-27 (2009).

45. Sibly, R.M. \& Calow, P. Ecological compensation - a complication for testing life history theory. J. Theor. Biol. 125, 177-186 (1987).

46. Van Valen, L. A new evolutionary law. Evolutionary Theory 1, 1-30 (1973). 
47. Tolkamp, B., Wall, E., Roehe, R., Newbold, J. \& Zaralis, K. Review of nutrient efficiency in different breeds of farm livestock. Report to DEFRA IF0183 (2010).

48. Hutchinson, G.E. Concluding remarks. Cold Spring Harbor Symposium Quant. Biology. 22, 415-427 (1957).

49. Hall, C.A.S., Stanford, J.A. \& Hauer, F.R. The distribution and abundance of organisms as a consequence of energy balances along multiple environmental gradients. Oikos 65, 377-390 (1992).

50. Hall, C.A.S. Energy Return on Investment: A unifying principle for Biology, Economics and sustainability (Springer, 2017).

\section{Additional information}

Reprints and permissions information is available at www.nature.com/reprints.

Correspondence should be addressed to J.H.B.

How to cite this article: Brown, J.H., Hall, C.A.S. \& Sibly, R.M. Equal fitness paradigm explained by a tradeoff between generation time and energy production rate. Nat. Ecol. Evol. ??

Publisher's note: Springer Nature remains neutral with regard to jurisdictional claims in published maps and institutional affiliations.

\section{SI Guide}

SI Table 1 contains the data of Fig. 2 as an Excel file with columns giving taxon, genus, species, dry body mass, temperature in ${ }^{\circ} \mathrm{C}$, uncorrected mortality rate, $\log _{10}$ dry body mass and $\log _{10}$ generation time at $20^{\circ} \mathrm{C}$, for 2026 species.

SI Table 2 contains the data of Fig. 4 as an Excel file with columns giving taxon, genus and species, dry body mass and ash-free energy content for 74 species. 